\title{
Efficacy and Safety of Glecaprevir/Pibrentasvir for Chronic Hepatitis C Patients: A Systematic Review and Meta-analysis
}

\author{
Hong-Qin $\mathrm{Xu}^{1}{ }^{1}$, Chun-Guang Wang ${ }^{2}$, Peng $\mathrm{Xiao}^{1}$ and Yan-Hang Gao*1 \\ ${ }^{1}$ Department of Hepatology, The First Hospital of Jilin University, Jilin University, Changchun, Jilin, China; ${ }^{2}$ Department of \\ Surgery, The Second Hospital of Jilin University, Changchun, Jilin, China
}

\begin{abstract}
Background and Aims: Glecaprevir/pibrentasvir is a pangenotypic regimen recently approved for the treatment of chronic hepatitis $\mathrm{C}$ virus (HCV) infection. The objective of the present review was to summarize the findings from clinical trials to understand how patient-related factors influence glecaprevir/pibrentasvir efficacy (sustained virologic response rates at 12 weeks' after treatment [referred to as SVR12]) and safety. Methods: Data from 21 phase III clinical trials were analyzed. Results: The integrated efficacy analysis included 4,817 patients. Findings showed $97.5 \%$ of all included patients with chronic HCV achieved SVR12 in the intention-to-treat population. SVR12 rate was $>95 \%$ across subgroups of interest. The integrated safety analysis included 4,015 patients. Findings showed that $64.1 \%$ of patients reported an adverse event, and $<0.1 \%$ of patients reported a serious adverse event related to glecaprevir/pibrentasvir. Conclusions: These results indicate that the 8 - or 12 -week glecaprevir/pibrentasvir treatment is effective for patients infected with HCV genotypes 1-6 without or with compensated cirrhosis, with good safety profiles, irrespective of treatmentexperience. Glecaprevir/pibrentasvir is a good option for patients with human immunodeficiency virus/HCV coinfection and comorbid HCV and severe renal impairment.

Citation of this article: $\mathrm{Xu} \mathrm{HQ}$, Wang CG, Xiao P, Gao YH. Efficacy and safety of glecaprevir/pibrentasvir for chronic hepatitis $C$ patients: A systematic review and meta-analysis. J Clin Transl Hepatol 2020;8(3):267-276. doi: 10.14218/ JCTH.2020.00047.
\end{abstract}

\section{Introduction}

World Health Organization estimates indicate that the global infection rate of hepatitis $\mathrm{C}$ virus ( $\mathrm{HCV}$ ) is $0.5-2.3 \%$ and that 71 million individuals have chronic hepatitis $C .{ }^{1}$ The clinical

Keywords: Hepatitis C virus; Chronic hepatitis C; Cirrhosis; Glecaprevir/ pibrentasvir; ABT-493/ABT-530; Meta-analysis; Clinical trials.

Abbreviations: $A E$, adverse event; $C I$, confidence interval; $D A A$, direct-acting antiviral; GT, genotype; HCV, hepatitis $C$ virus; HIV, human immunodeficiency virus; IFN, interferon; ITT: intention-to-treat; PEG-IFN, pegylated interferon; mITT, modified intention-to-treat; MINORS, methodological index for nonrandomized studies; SAE, serious adverse event; SVR12, sustained virologic response at 12 weeks; SOF/VEL/VOX, sofosbuvir/velpatasvir/voxilaprevir; SRI, severe renal impairment.

Received: 17 May 2020; Revised: 10 August 2020; Accepted: 12 August 2020 *Correspondence to: Yan-Hang Gao, Department of Hepatology, The First Hospital of Jilin University, Jilin University, No. 71, Xinmin Street, Changchun, Jilin 130021, China. Tel: +86-431-81875121, E-mail: yanhang@mail.jlu.edu.cn and economic burdens attributable to HCV infection are substantial, as an estimated 500,000 people die annually from liver disease caused by $\mathrm{HCV}^{2}$ Interferon (IFN)-free, directacting antiviral (DAA) agents are standard-of-care for chronic hepatitis $C$, achieving a very high sustained virologic response at 12 weeks (SVR12). Glecaprevir/pibrentasvir is a pangenotypic regimen approved to treat chronic HCV that contains two antiviral drugs: glecaprevir, an NS3/4A protease inhibitor, and pibrentasvir, an HCV NS5A inhibitor. The glecaprevir/pibrentasvir global clinical development program included patients with all HCV genotypes (GTs), different stages of fibrosis, presence/absence of cirrhosis, human immunodeficiency virus (HIV) coinfection, and those who did not respond to prior DAA-based treatment. The objective of the present review was to describe the findings from glecaprevir/pibrentasvir clinical trials to understand how patientrelated factors influence efficacy and safety.

\section{Methods}

\section{Search and review strategy}

The Embase, PubMed, and Cochrane databases and the clinical trial registration website (ClinicalTrials.gov) were searched from inception to March 2020, using the following search terms: (glecaprevir and pibrentasvir) OR (glecaprevir/ pibrentasvir) OR (glecaprevir plus pibrentasvir) OR (ABT-493/ ABT-530) OR (maviret) OR (mavyret) AND ((HCV) OR (hepatitis C)) AND (clinical trial).

\section{Selection criteria}

Clinical trials reporting the efficacy and safety of glecaprevir/ pibrentasvir for HCV infection were included in this review. Exclusion criteria were: 1 ) studies that did not provide SVR12 data or in which SVR12 could not be calculated; 2) studies that investigated glecaprevir and/or pibrentasvir pharmacokinetics in healthy subjects; 3) real-world cohort studies, case reports, or studies that grouped patients administered glecaprevir/pibrentasvir with patients administered other regimens; or 4) book chapters, abstracts, conference papers, reviews, editorials, or letters.

\section{Data extraction}

The meta-analysis was conducted with $\mathrm{R}$ software (meta_4.11-0 package). Event rates were transformed to proportions and corresponding $95 \%$ confidence intervals (CIs) using the double arcsine method. Heterogeneity of 
Xu H.Q. et al: MAVYRET for HCV infection

data was evaluated using the $I^{2}$ statistic and Cochrane Q test. If significant heterogeneity $\left(I^{2}>50 \%, P<0.1\right)$ existed between studies, data were evaluated with a random-effects model; alternatively, a fixed-effects model was used. Efficacy (SVR12) and safety of glecaprevir/pibrentasvir for chronic HCV infection were investigated. Glecaprevir/pibrentasvir efficacy was explored in subgroups stratified by HCV genotype, presence or absence of cirrhosis, treatment history, treatment duration, and comorbidities. Glecaprevir/pibrentasvir safety was stratified by any adverse event (AE), serious adverse events (SAEs), and drug-related SAEs.

\section{Results}

\section{Literature selection and basic information}

Overall, 25 studies ( 21 publications and 4 unpublished clinical trials; ClinicalTrials.gov Nos. NCT02707952 substudy $1,{ }^{3}$ NCT0 $2723084,{ }^{4}$ NCT02604017, ${ }^{5}$ NCT02640482,
NCT02640157, ${ }^{5}$ NCT02636595, $6,7 \quad$ NCT02966795, 8 NCT02642432, ${ }^{9}$ NCT02738138, ${ }^{10}$ NCT02651194, ${ }^{11}$ NCT03069365, ${ }^{12}$ NCT03089944, ${ }^{13}$ NCT02446717, ${ }^{14,15}$ NCT02692703, ${ }^{16}$ NCT03117569, ${ }^{17}$ NCT02243293, $6,18,19$ NCT03222583, ${ }^{20}$ NCT03235349, ${ }^{21}$ NCT03092375, ${ }^{22}$ NCT03212521, ${ }^{23}$ NCT03219216 ${ }^{24}$ ) were included in this review. A flow chart of the study selection is presented in Fig. 1. The trials included 4817 patients with chronic HCV of genotypes (GTs) 1-6, in the presence or absence of compensated cirrhosis, HIV, severe renal impairment (SRI), or prior treatment with DAAs. Patients in the included studies were administered glecaprevir (300 mg) and pibrentasvir (120 mg). Characteristics of the included trials are provided in Table 1.

\section{Clinical efficacy: SVR12 rates in all integrated patients}

SVR12 rates in all HCV genotypes were described in 21 phase III clinical trials. SVR12 rates in patients administered glecaprevir/pibrentasvir ( $n=4817$ patients) ranged from $90.1 \%$

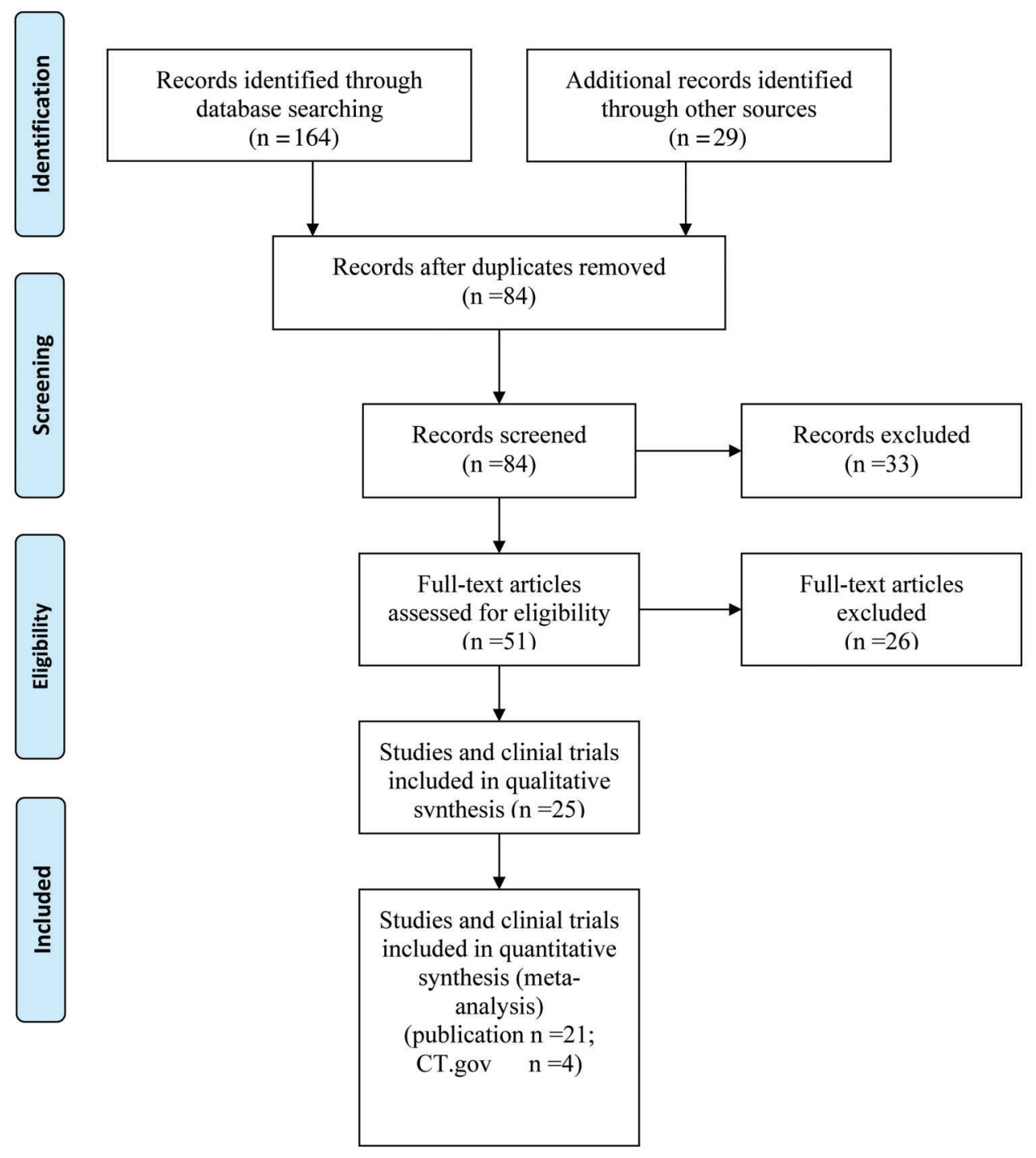

Fig. 1. Flow chart of the search strategy. 


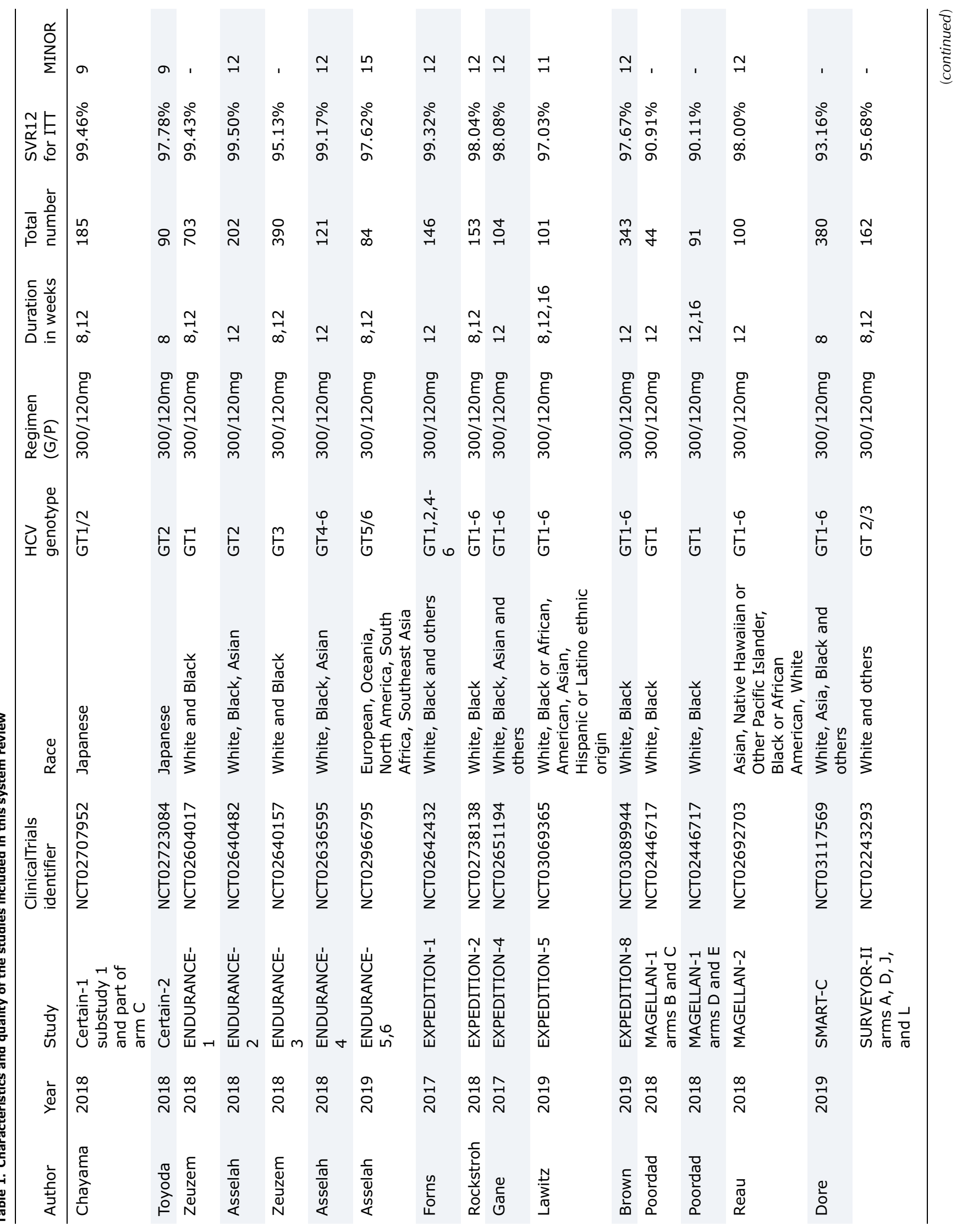




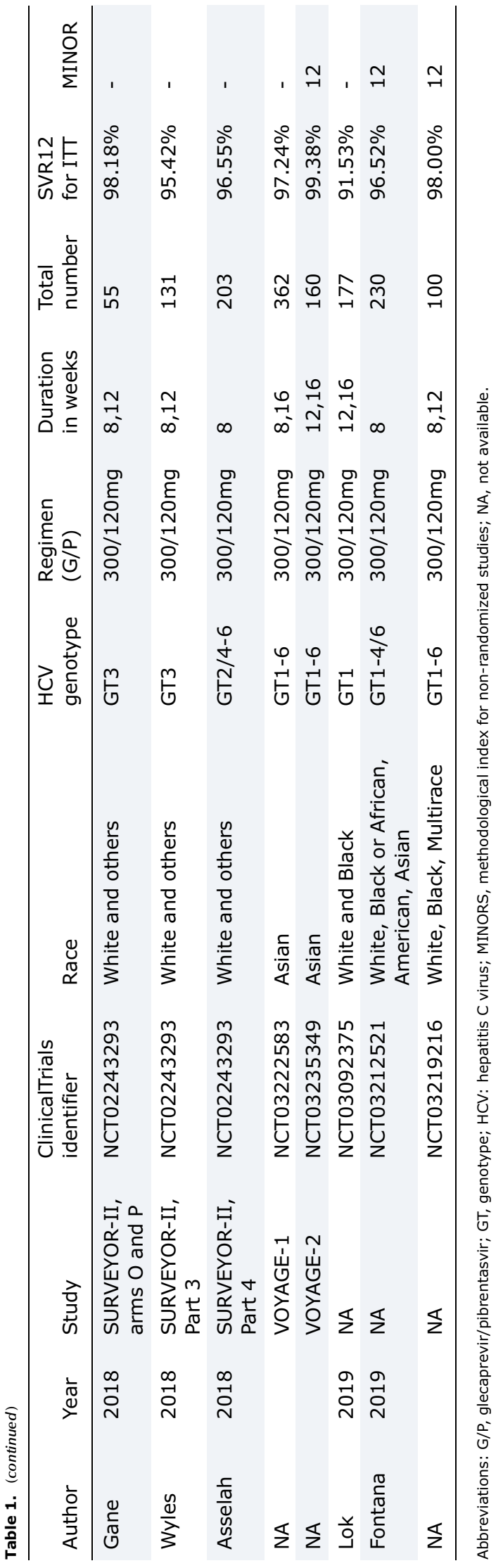

to $99.8 \%$ in the intention-to-treat (ITT) populations in the individual trials. In the integrated efficacy analysis, $97.5 \%$ (95\% CI $96.4-98.2 \%, I^{2}=75 \%$ ) of the patients in the ITT populations administered glecaprevir/pibrentasvir achieved SVR12 (Fig. 2). Data from the modified ( $\mathrm{mITT}$ ) populations were described in 11 trials. SVR12 rates in patients administered glecaprevir/pibrentasvir ( $n=1765$ patients) ranged from $95.2 \%$ to $100 \%$ in the mITT populations in the individual trials. In the integrated efficacy analysis, $99.7 \%$ (95\% CI $98.9-100 \%, I^{2}=72 \%$ ) of the patients in the mITT populations administered glecaprevir/ pibrentasvir achieved SVR12 (Fig. 3).

\section{SVR12 rates in all HCV genotypes}

SVR12 rates by GT were described in 13 phase III clinical trials (ClinicalTrials.gov Nos. NCT02707952, ${ }^{3}$ NCT02723084, ${ }^{4}$ NCT02604017, ${ }^{5} \quad$ NCT02640482, ${ }^{6}$ NCT02640157, ${ }^{5}$ NCT02636595, $6,7 \quad$ NCT02966795, ${ }^{8} \quad$ NCT02642432, 9 NCT03089944, ${ }^{13}$ NCT02446717, ${ }^{14,15}$ NCT02243293, $, 18,19$ NCT03222583, ${ }^{20}$ NCT03092375, ${ }^{22}$ NCT03212521 ${ }^{23}$ ), including 3585 patients administered glecaprevir/pibrentasvir. Of these, 1740 (48.5\%) patients were infected with HCV GT 1, $765(21.3 \%)$ patients were infected with HCV GT 2, 758 (21.1\%) patients were infected with HCV GT 3, and 322 (9.1\%) patients were infected with HCV GTs 4-6. In the integrated efficacy analysis, $97.6 \%$ (95\% CI: 95.4-99.1\%), 99.0\% (95\% CI: $97.9-99.7 \%), \quad 95.3 \%$ (95\% CI: $93.5-$ $96.6 \%), 100 \%$ (95\% CI: $100-100 \%), 96.6 \%$ (95\% CI: 86.4-100\%), and $99.0 \%$ (95\% CI: $95.7-100 \%)$ of patients with HCV GT 1, 2, 3, 4, 5 and 6, respectively, achieved SVR12 (Fig. 4a). These data indicate the SVR12 rate was lower in the subpopulation of patients with HCV GT 3.

\section{SVR12 rates in patients with compensated cirrhosis or without cirrhosis}

SVR12 rates in patients without cirrhosis were described in 14 phase III clinical trials (NCT02707952, ${ }^{3}$ NCT02723084, ${ }^{4}$ NCT02604017, ${ }^{5} \quad$ NCT02640482, ${ }^{6}$ NCT02640157, ${ }^{5}$ NCT02636595, 6,7 NCT02966795, 8 NCT02738138, 10 NCT02446717, ${ }^{14,15}$ NCT03117569, ${ }^{17}$ NCT02243293, ${ }^{6,18,19}$ NCT03222583, ${ }^{20}$ NCT03092375, ${ }^{22}$ NCT03212521 ${ }^{23}$ ), including 3400 patients administered glecaprevir/pibrentasvir. SVR12 rates in patients with compensated cirrhosis were described in 8 phase III clinical trials (NCT02707952, ${ }^{3}$ NCT02966795, ${ }^{8} \quad$ NCT02642432, ${ }^{9}$ NCT02738138, ${ }^{10}$ NCT03089944,' 13 NCT02243293, 6, 18,19 NCT03235349, ${ }^{21}$ NCT03092375 22) including 923 patients administered glecaprevir/pibrentasvir. In the integrated efficacy analysis, 97.3\% (95\% CI: $95.8-98.5 \%$ ) and $98.0 \%$ (95\% CI: $95.9-$ $99.3 \%$ ) of the patients in the ITT populations without or with compensated cirrhosis, respectively, achieved SVR12 (Fig. 4b). SVR12 rates between the two groups were not significantly different.

\section{SVR12 rates by treatment history}

SVR12 rates in DAA-naïve patients were described in 9 phase III clinical trials (NCT02707952, ${ }^{3}$ NCT02723084, ${ }^{4}$ NCT02604017, ${ }^{5}$ NCT02640482, ${ }^{6} \quad$ NCT02640157, ${ }^{5}$ NCT03089944, ${ }^{13}$ NCT03117569, ${ }^{17}$ NCT03212521, ${ }^{23}$ NCT03219216 ${ }^{24}$ ), including 2,535 patients administered glecaprevir/pibrentasvir. SVR12 rates in DAA-experienced patients were described in 4 phase III clinical trials (NCT02707952, ${ }^{3}$ NCT02604017, 
Xu H.Q. et al: MAVYRET for HCV infection

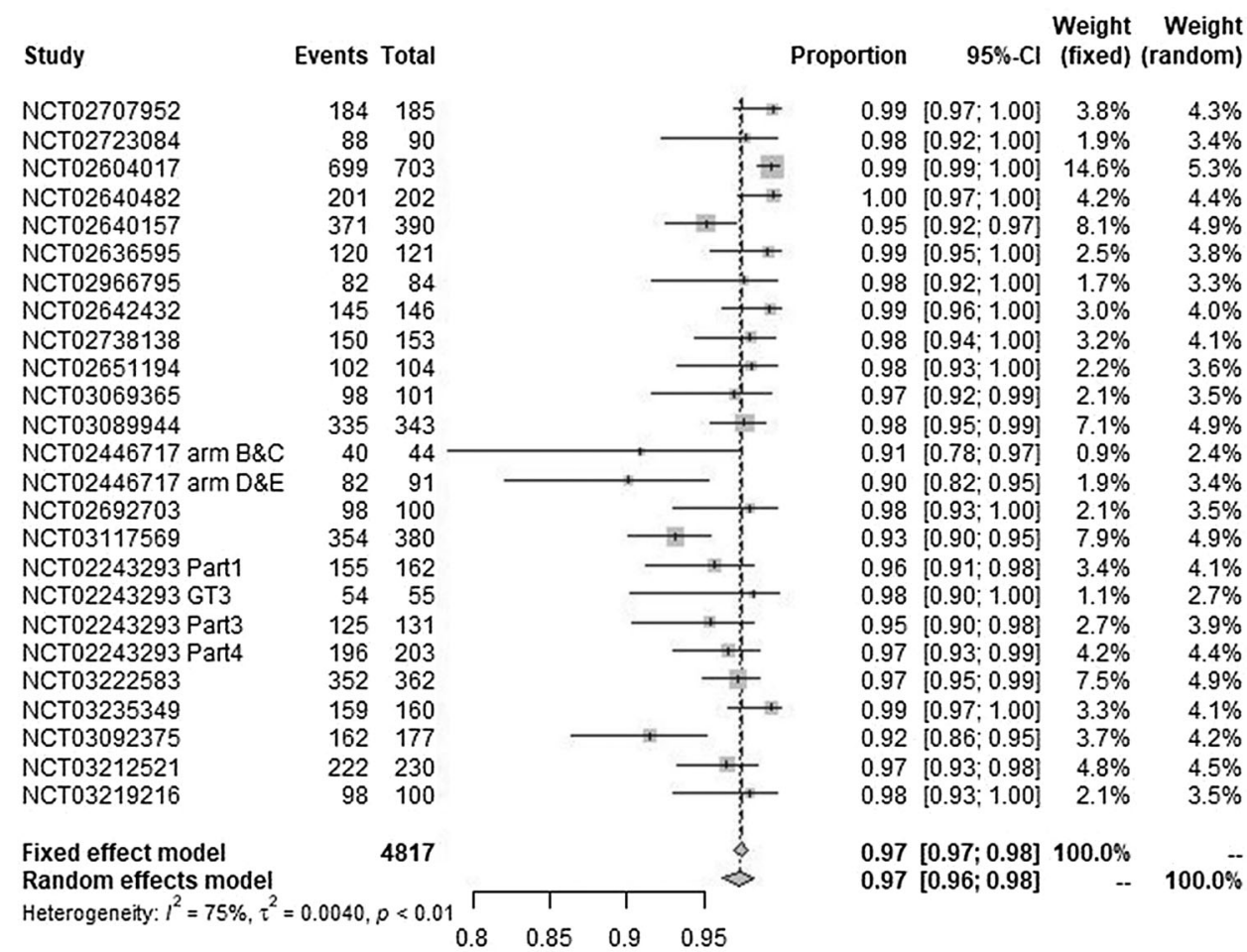

Fig. 2. Forest plots showing SVR12 rates for glecaprevir/pibrentasvir in the ITT population $(n=4817)$.

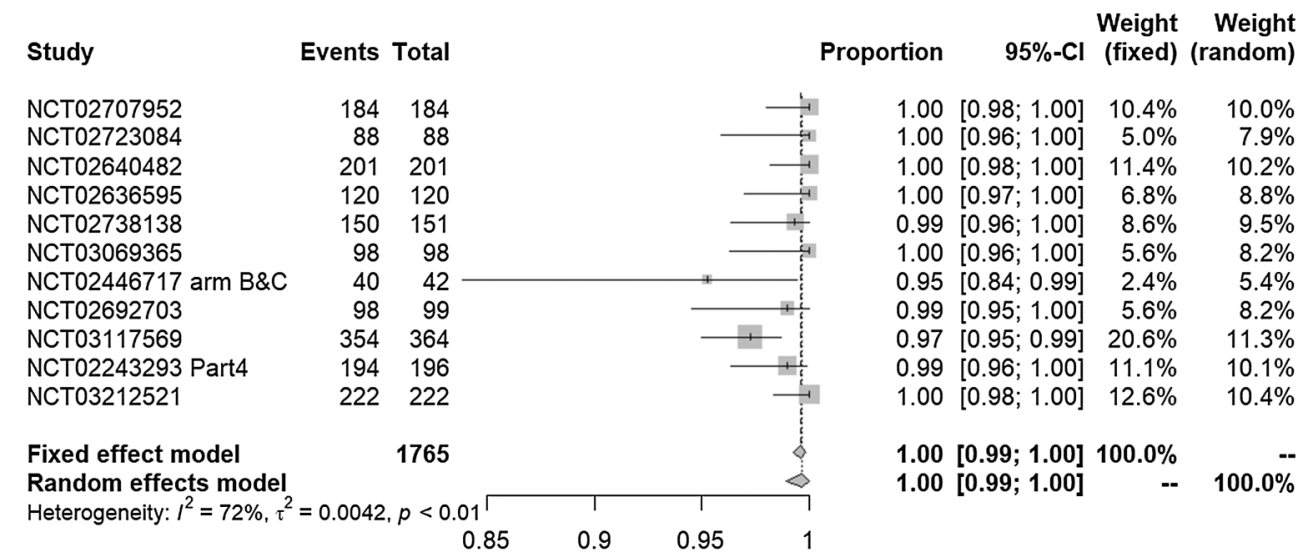

Fig. 3. Forest plots showing SVR12 rates for glecaprevir/pibrentasvir in the mITT population $(n=1765)$.

NCT02640482, ${ }^{6}$ NCT0309237522), including 219 patients administered glecaprevir/pibrentasvir. In the integrated efficacy analysis, $97.7 \%$ (95\% CI: $95.9-99.0 \%$ ) and $96.2 \%$ (95\% CI: $92.1-99.1 \%$ ) of DAA-naïve and DAA-experienced patients, respectively, achieved SVR12 (Fig. 4C).

\section{Duration of treatment}

In 2017, 8-week glecaprevir/pibrentasvir was approved in the USA and Europe for patients infected with all HCV GTs without cirrhosis. Pooled data from 13 phase III clinical trials (NCT02604017, 5 NCT02640482, ${ }^{6} \quad$ NCT02640157, 5 NCT02636595, ${ }^{6,7}$ NCT02642432, ${ }^{9}$ NCT02651194, ${ }^{11}$ NCT03089944, ${ }^{13}$ NCT02446717, ${ }^{14,15}$ NCT02692703, ${ }^{16}$ NCT03117569, 17 NCT02243293, 6,18,19 NCT03092375, 22
NCT03212521 23 ) were included in an integrated efficacy analysis of 2818 patients infected with HCV GTs 1-6 administered glecaprevir/pibrentasvir for 8,12 or 16 weeks. Findings showed that $97.5 \%$ (95\% CI: $95.8-98.8 \%$ ) and $97.8 \%$ (95\% CI: $95.9-99.2 \%$ ) of patients in the ITT population administered 8 or 12 weeks of glecaprevir/pibrentasvir, respectively, achieved SVR12 (Fig. 4d).

\section{HIV/HCV coinfection}

SVR12 rates in patients with HCV and no HIV coinfection were described in 20 phase III clinical trials, including 4631 patients administered glecaprevir/pibrentasvir. In the integrated efficacy analysis, $97.7 \%$ (95\% CI: $96.5-98.5 \%)$ of these patients achieved SVR12. SVR12 rates in patients with 
a

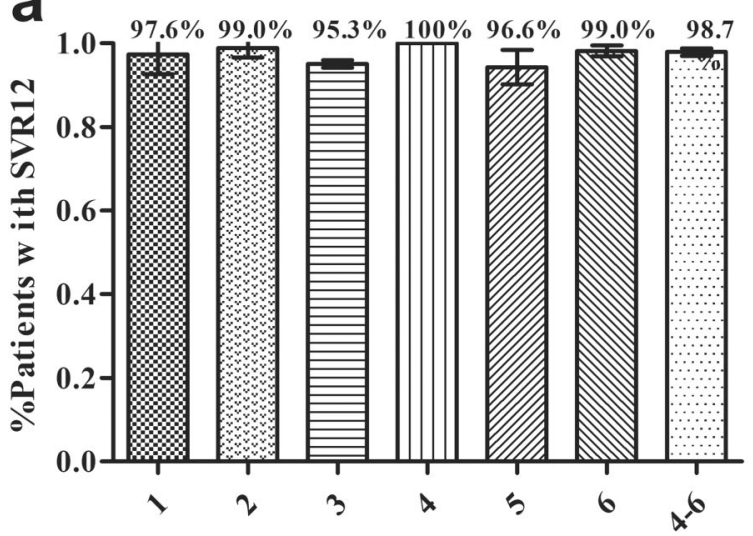

HCV genotypes

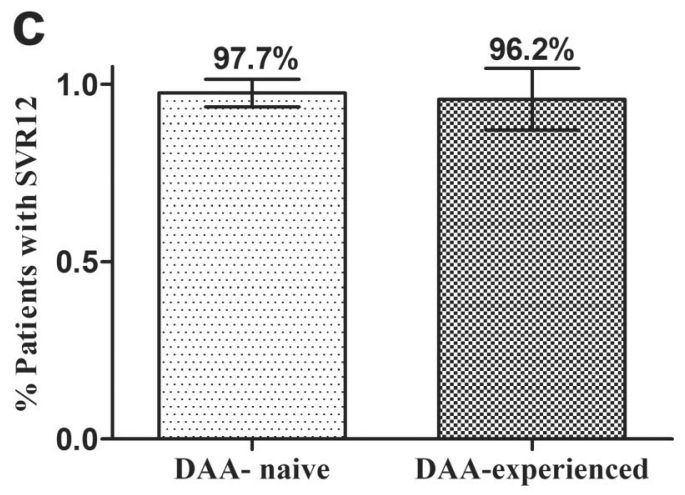

e

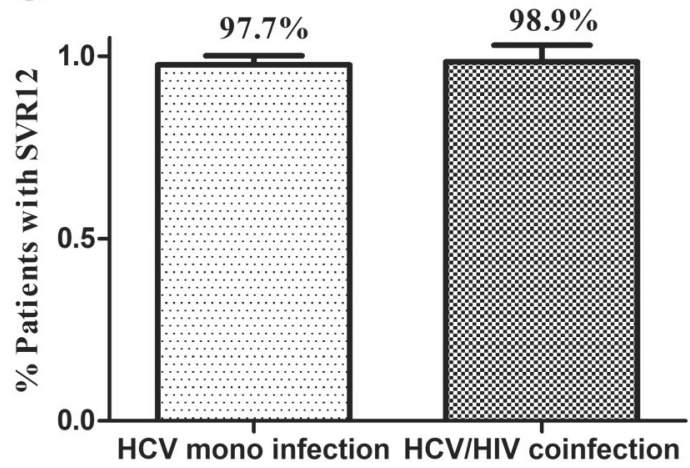

b

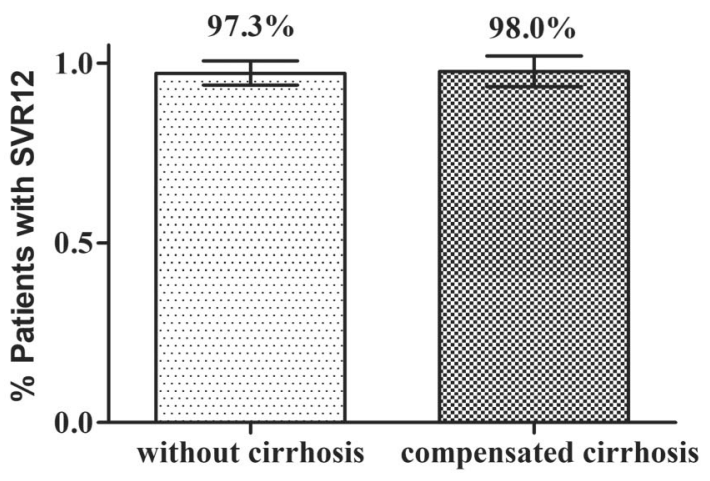

d
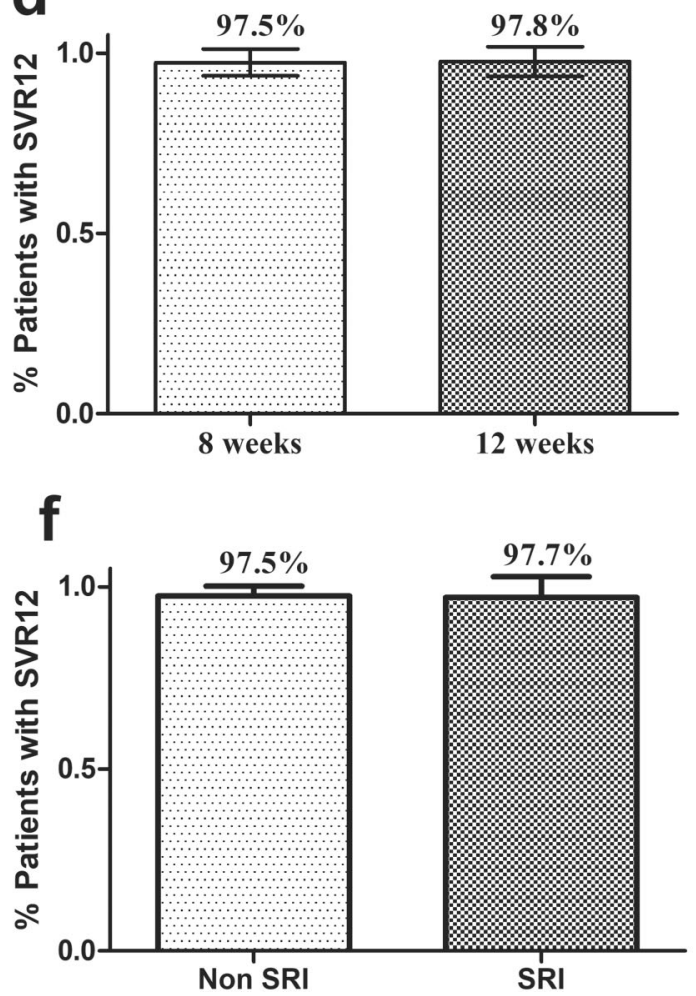

Fig. 4. Integrated efficacy analysis (SVR12) in the ITT population.

(a) GTs. (b) With/without cirrhosis. (c) Treatment history. (d) Treatment duration. (e) With/without HIV co-infection, (f) With/without SRI.

HCV and HIV coinfection were described in 2 phase III clinical trials, including 186 patients administered glecaprevir/ pibrentasvir. In the integrated efficacy analysis, $98.9 \%$ (95\% CI: $96.4-100 \%$ ) of these patients achieved SVR12 (Fig. 4e).

\section{Comorbid HCV and SRI}

SVR12 rates in patients with HCV and SRI were described in 19 phase III clinical trials, including 4612 patients administered glecaprevir/pibrentasvir. In the integrated efficacy analysis, $97.5 \%$ (95\% CI: $96.3-98.5 \%)$ of these patients achieved SVR12. In 3 trials, 217 patients with SRI achieved high rates of SVR12 (97.7\%, 95\% CI: 94.5-99.0\%) (Fig. 4f).

\section{Safety}

Safety data were available for 4015 patients (safety population; 18 cohorts) administered glecaprevir/pibrentasvir. Of these, $2420(64.1 \%)$ patients reported an AE and 125 patients $(3.32 \%)$ reported an SAE. In the integrated safety analysis, the most frequently reported AEs were fatigue $(13.66 \%)$, headache $(12.81 \%)$ and nausea $(7.40 \%)$ (Table 2). Other AEs included diarrhea, nasopharyngitis, 
insomnia, and dizziness. Three $(<0.1 \%)$ patients reported an SAE related to glecaprevir/pibrentasvir. Of these, $2(1 \%)$ patients reported angioedema that led to glecaprevir/pibrentasvir discontinuation on day 8 and 15, respectively; subsequently, the angioedema resolved within 7 and 3 days, respectively. Both patients were Black or African American, had a history of drug use, were receiving an angiotensin-converting enzyme inhibitor, and had an HIV coinfection. In ENDURANCE 4 (NCT02636595), 1 patient discontinued glecaprevir/pibrentasvir on day 12 due to a transient ischemic attack that was considered related to glecaprevir/pibrentasvir. $^{6,7}$ In ENDURANCE 2 (NCT02640482), the incidence of AEs was similar in the placebo and active glecaprevir/pibrentasvir arms. ${ }^{6}$

\section{Discussion}

This meta-analysis of data from 25 clinical trials revealed that 8 or 12 weeks of glecaprevir/pibrentasvir treatment is a safe and effective pangenotypic treatment option for patients with chronic HCV infection. Among the included trials, only 1765/ 4817 patients were included in the MITT population, as 9 trials did not report these data. Findings showed that $\geq 97.0 \%$ and $95.3 \%$ of patients with HCV GTs $1,2,4-6$ and HCV GT 3 achieved SVR12, respectively.

HCV GT 3 infection is difficult to treat, as disease progression is rapid and the complication rate, including for hepatocellular carcinoma, is higher than in other GTs. SVR12 rates range from $92-100 \%$ in treatment-naïve patients with HCV GT 3 , in the presence or absence of compensated cirrhosis, administered most DAA-based therapies. ${ }^{25}$ Glecaprevir/pibrentasvir and sofosbuvir/velpatasvir/ voxilaprevir (commonly known as SOF/VEL/VOX) achieve SVR12 rates approaching $96 \%$ in treatment-experienced patients with HCV GT 3. ${ }^{25}$ In ENDURANCE-3, SVR12 rates were $95 \%(95 \%$ CI: $93-98 \% ; n=222 / 233)$ and $97 \%(95 \%$ CI: $93-99.9 \% ; n=111 / 115)$ in treatment-naïve patients with HCV GT 3 and no cirrhosis administered glecaprevir/pibrentasvir or sofosbuvir/daclatasvir, respectively, for 12 weeks. $^{5}$

Table 2. Rate of AEs and SAEs of G/P for patients with HCV genotypes 1-6 infection

\begin{tabular}{|c|c|c|c|c|}
\hline Event & Total & $n$ & Rate & $95 \% \mathrm{CI}$ \\
\hline Any $\mathrm{AE}, n(\%)$ & 4015 & 2420 & $64.13 \%$ & $\begin{array}{l}59.2- \\
68.9 \%\end{array}$ \\
\hline Serious $A E, n(\%)$ & 4015 & 125 & $3.32 \%$ & $\begin{array}{l}1.96- \\
4.99 \%\end{array}$ \\
\hline $\begin{array}{l}\text { DAA-related } \\
\text { serious } \mathrm{AE}, n(\%)\end{array}$ & 1732 & 3 & $0.03 \%$ & $\begin{array}{l}0- \\
0.26 \%\end{array}$ \\
\hline \multicolumn{5}{|l|}{$\begin{array}{l}\text { AEs occurring } \\
\text { mostly }\end{array}$} \\
\hline $\begin{array}{l}\text { Fatigue, } \\
n(\%)\end{array}$ & 1541 & 222 & $13.66 \%$ & $\begin{array}{l}10.03- \\
18.34 \%\end{array}$ \\
\hline $\begin{array}{l}\text { Headache, } \\
n(\%)\end{array}$ & 1631 & 217 & $12.81 \%$ & $\begin{array}{l}9.77- \\
16.18 \%\end{array}$ \\
\hline $\begin{array}{l}\text { Nausea, } \\
n(\%)\end{array}$ & 1401 & 113 & $7.40 \%$ & $\begin{array}{l}5.24- \\
9.56 \%\end{array}$ \\
\hline
\end{tabular}

Abbreviations: $A E$, adverse event; $C I$, confidence interval; $G / P$, glecaprevir/ pibrentasvir; SAE, serious adverse event.
The 12-week glecaprevir/pibrentasvir regimen was noninferior to the 12-week sofosbuvir/daclatasvir regimen. In fact, real world data have shown that patients with HCV GT $3 \mathrm{~b}$ achieved lower SVR12 rates $(33 \%[2 / 6]-50.0 \%[2 / 4])$ than patients with HCV GT $3 a$ with the 12 -week regimen. ${ }^{26,27}$

In the present analysis, response rates to glecaprevir/ pibrentasvir in the ITT populations were not affected by the presence (SVR12: 97.3\% [95\% CI: 95.8-98.5\%])/absence (SVR12: $98.0 \%$ [95\% CI: 95.9-99.3\%]) of compensated cirrhosis. The SVR12 rate in patients without cirrhosis may have been affected by inclusion of the trial by the Smart-C Study Group, which included 380 patients with HCV without cirrhosis who were administered glecaprevir/pibrentasvir. The SVR12 rate in the ITT population was $92 \%$, possibly because 14 patients were lost to follow up. ${ }^{17}$ Consistent with our findings, SVR12 rates were not influenced by the presence or absence of cirrhosis in real world studies. ${ }^{28,29}$ Currently, glecaprevir/pibrentasvir is not recommended for patients with decompensated cirrhosis (Child-Pugh B and Child-Pugh C) due to elevated drug exposures. Pharmacokinetic studies of glecaprevir $300 \mathrm{mg} /$ pibrentasvir $120 \mathrm{mg}$ showed a 2.0-fold to 11-fold increase in glecaprevir AUC in HCV-negative subjects with moderate or severe hepatic impairment compared to normal subjects. ${ }^{30-32}$

The European Association for the Study of the Liver and the American Association for the Study of Liver Diseases recommend 12 weeks of SOF/VEL/VOX as the first-line retreatment option for patients with $\mathrm{HCV}$ that have failed DAA regimens. EASL 2018 guidelines recommend a 12 week SOF+ glecaprevir/pibrentasvir regimen as a second-line treatment option. This recommendation is based on evidence from MAGELLAN-1 (part 2), which reported SVR12 rates of $95 \%$ in patients with HCV GT 1 and prior DAA treatment who had received glecaprevir/pibrentasvir in the presence or absence of IFN (mITT population). ${ }^{14,33}$ In the present analysis, response rates to glecaprevir/pibrentasvir in the ITT populations were not affected by treatment history (DAA-naïve SVR12: $97.7 \%$ [95\% CI: 95.9-99.0\%]; DAA-experienced SVR12 $96.2 \%$ [95\% CI: 92.1-99.1\%]). In contrast to our findings, a previous meta-analysis found a lower SVR12 rate for DAA-experienced vs. DAA-naïve patients administered glecaprevir/pibrentasvir (96.1\% vs. 99.7\%). ${ }^{34}$ These disparate results may be explained by our inclusion of data from the Smart-C Study Group, where the patients lost to follow-up may have influenced the results.

SVR12 rates may be lower in DAA-experienced patients, as these patients have a higher probability of HCV GT 3 infection, advanced liver disease, and DAA-class-specific resistanceassociated substitutions. Most recommendations suggest resistance-associated substitution testing should guide retreatment. ${ }^{35,36}$ One study showed that glecaprevir/pibrentasvir was effective for DAA-experienced patients with HCV who did not achieve an SVR, except for patients with HCV GT $1 b$ who were carrying the NS5A-P32del mutation. ${ }^{37}$ Another study (NCT03092375) showed that the overall SVR 12 was $95 \%$ and that only patients with GT1a infection and not GT1b infection failed treatment with an NS5A inhibitor plus sofosbuvir. Thus, the authors recommend not testing for RAS before retreatment. ${ }^{22}$ In another report, retreatment with 12 weeks of glecaprevir/pibrentasvir was successful in a hemodialysis patient with HCV GT 2a who had previously failed 8 weeks of glecaprevir/pibrentasvir therapy. ${ }^{38}$

In terms of glecaprevir and pibrentasvir treatment duration, the present analysis found SVR12 rates in patients with 
HCV GT 1-6 administered glecaprevir/ pibrentasvir for 8 weeks (97.5\% [95\% CI: $95.8-98.8 \%$ ]) or 12 weeks (97.8\% [95\% CI: 95.9-99.2\%]) were not significantly different. Consistent with this, an integrated analysis of 9 phase II and III clinical trials in 2041 patients with HCV GT 1-6 and no cirrhosis reported similar SVR12 rates in the ITT population for the $8-(98 \%)$ or 12 -week (99\%) glecaprevir/pibrentasvir regimens. ${ }^{39}$ These findings are also supported by real world data (SVR12: 8-week vs. 12- or 16-week; 99.2\% [95\% CI: $98.5-99.9 \%]$ vs. $100 \% ; P=1.00) .{ }^{40}$ The present review did not include an integrated efficacy analysis of SVR12 rates after 16 weeks of glecaprevir/pibrentasvir treatment, as only 2 clinical trials reported these data. In 1 randomized trial, $>90 \%$ patients with HCV GT 1 (including those with compensated cirrhosis) who were previously administered sofosbuvir plus an NS5A inhibitor achieved SVR12 following 16 weeks of glecaprevir/pibrentasvir treatment. In the same randomized study, patients administered 12 weeks of glecaprevir/pibrentasvir had lower SVR12 rates $(88.9 \%){ }^{22}$ In MAGELLAN-1, $89 \%$ and $91 \%$ of patients administered 12 and 16 weeks of glecaprevir/pibrentasvir treatment achieved SVR12, respectively. ${ }^{15}$

Glecaprevir/pibrentasvir treatment is effective for hepatitis $\mathrm{C}$ and comorbidities, including HIV and SRI. Patients with HIV/HCV coinfection have a higher probability of developing cirrhosis than patients infected with HCV alone. In the present review, $97.8 \%$ of patients with HIV/HCV achieved SVR12 with glecaprevir/pibrentasvir. Specifically, in EXPEDITION-2, 99\% of patients with HCV GT 1-6/HIV-1 in the presence or absence of compensated cirrhosis in the mITT population achieved SVR 12 with 8 or 12 weeks glecaprevir/pibrentasvir treatment. There were no virologic failures among patients with no cirrhosis administered 8 weeks of glecaprevir/pibrentasvir. One HCV GT 3 treatment-naive patient with compensated cirrhosis (12-week treatment arm) had on-treatment virologic breakthrough at week 8 and did not achieve SVR12. ${ }^{10}$ In 3 Japanese patients with HCV GT 3/HIV coinfection and hemophilia, glecaprevir/pibrentasvir was safe and effective. ${ }^{2}$ In patients with acute HCV and HIV, treatment with glecaprevir/pibrentasvir according to current recommendations (2019) for chronic HCV achieved SVR12 rates of $100 \% .41$

Drug-drug interactions between glecaprevir/pibrentasvir and antiretrovirals used to treat HIV (integrase inhibitors, nonnucleoside reverse transcriptase, inhibitors, nucleoside/ tide analogues, pharmacokinetic enhancers, and protease inhibitors) have been characterized in 7 phase 1 clinical trials. ${ }^{42}$ Glecaprevir/pibrentasvir exposure levels were significantly increased by ritonavir-boosted protease (which inhibit CYP3A, P-glycoprotein, and breast cancer resistant protein) inhibitors and significantly decreased by efavirenz (CYP3A4 inducer), as glecaprevir is a substrate of P-glycoprotein, breast cancer resistant protein and OATP1B1/3 and pibrentasvir is a substrate of P-glycoprotein and/or breast cancer resistant protein. Metabolism of glecaprevir/pibrentasvir is minimal, and glecaprevir/pibrentasvir may inhibit P-glycoprotein, breast cancer resistant protein, and OATP1B1/3 transporters and weakly inhibit CYP3A. ${ }^{43}$ Atazanavir is contraindicated in patients administered glecaprevir/pibrentasvir, and boosted protease inhibitors or efavirenz should not be used. Other antiretroviral agents and bictegravir-based HIV regimens are not expected to interact with glecaprevir/ pibrentasvir.

Glecaprevir/pibrentasvir is used in patients with HCV and an estimated glomerular filtration rate of $<30 \mathrm{~mL} \mathrm{~min} / 1.73$ $\mathrm{m}^{2}$. Findings from the present analysis showed high SVR12 rates in patients with HCV and SRI administered glecaprevir/ pibrentasvir. No patients experienced virologic failure or virologic relapse. ${ }^{13,14}$

Phase 1 trials support the safety of glecaprevir/pibrentasvir for HCV and SRI. In healthy subjects, glecaprevir and pibrentasvir are eliminated through the fecal-biliary route, with minor ( $<1 \%$ at clinical doses) renal clearance. ${ }^{44}$ Exposure levels of glecaprevir/pibrentasvir are not significantly affected by renal impairment or dialysis, indicating that patients with HCV and renal impairment may be administered glecaprevir/pibrentasvir without the need for dose adjustment or change in treatment duration. Consistent with this, real world data show $96 \%-100 \%$ of patients with HCV and SRI administered glecaprevir/pibrentasvir according to the product label achieved SVR12. ${ }^{45}$

The present analysis revealed that glecaprevir/pibrentasvir was well tolerated in patients with chronic HCV infection. Among the 4105 patients included in the safety evaluations, only 3 patients reported a drug-related SAE. The most frequent $A E s$ were fatigue, headache, and nausea. The incidence and severity of AEs and SAEs in patients randomized to glecaprevir/pibrentasvir or matching placebo were similar. ${ }^{6}$ Safety was similar in patients with HCV without cirrhosis treated for 8 or 12 weeks. The incidence of AEs and SAEs was higher in patients with HCV infection who underwent a liver or kidney transplant (MAGELLAN-2; NCT02692703), where 85 patients $(85 \%)$ reported an AE and $8(8 \%)$ patients reported an SAE, $2(2 \%)$ of which (sinusitis and abnormal hepatic function) were considered to be related to glecaprevir/pibrentasvir. ${ }^{16}$ As liver function may change during treatment with glecaprevir/pibrentasvir, the international normalized ratio values should be monitored closely.

Clinically, special populations, such as patients with chronic HCV who have had hepatic or renal transplants, the elderly, and the pediatric population, may be administered glecaprevir/pibrentasvir. In MAGELLAN-2 (NCT02692703), $98 \%$ of patients who had received primary liver or kidney transplants administered glecaprevir/pibrentasvir achieved SVR12 (98\%; 95\% CI: 95.3-100\%) (ITT analysis). The SVR12 rate was $99 \%(98 / 99 ; 95 \% \mathrm{CI}: 97-100 \%)$ in the mITT analysis, which excluded patients who failed to achieve SVR12 for nonvirologic reasons. Real world data confirm that 8- or 12-weeks of glecaprevir/pibrentasvir is effective in patients with liver transplantation and recurring $\mathrm{HCV}$, including difficult-to-treat populations, such as those with severe renal impairment, DAA treatment experience, cirrhosis, or jaundice after liver transplantation. ${ }^{46}$ Glecaprevir/pibrentasvir is also effective for patients aged $\geq 65$ years or $<18$ years. ${ }^{47,48}$ Although pharmacokinetic studies show glecaprevir/pibrentasvir exposure levels in adults and children are comparable, the safety and efficacy of glecaprevir/pibrentasvir in children aged I-12 years has not been established. However, in 1 study, a girl aged 10 years and 8 months achieved SVR12 with no AEs after 8 weeks of glecaprevir/ pibrentasvir treatment. ${ }^{49}$

This study was limited by high heterogeneity between clinical trials. Although the SVR12 rates in the ITT populations in the individual trials were all $>90 \%$, there was evidence of high heterogeneity between trials (SVR 12 rates: ITT population, $I^{2}=75 \%$; mITT population, $I^{2}=72 \%$ ). Further studies are required to understand the source of this heterogeneity but it is likely due to the inclusion of various trials that 
reported data on patients with different GTs and comorbidities in this meta-analysis. Despite the high heterogeneity between trials, the overall SVR12 rate for the ITT population $(97.5 \%)$ was consistent with a previously published integrated analysis $(97.8 \%) .{ }^{34}$

\section{Conclusions}

This integrated efficacy and safety analysis of glecaprevir/ pibrentasvir confirmed that 8- or 12-weeks of glecaprevir/ pibrentasvir treatment is effective for HCV GTs 1-6, including in patients with treatment-experience or compensated cirrhosis. Glecaprevir/pibrentasvir is a good option for patients with HIV/HCV or comorbid SRI.

\section{Funding}

This work was sponsored by the National Natural Science Foundation of China (Grant Nos. 81972265, 81700534), the National Key Research Plan "Precision Medicine Research" Key Project (2017YFC0908103, 2017YFC0908104, 2018ZX10302206), the National Science and Technology Major Project (2017ZX10202202), the JLU Norman Bethune Research Plan (2018B32), the Program for JLU Science and Technology Innovative Research Team (2017TD-08) and the Fundamental Research Funds for the Central Universities.

\section{Conflict of interest}

The authors have no conflict of interests related to this publication.

\section{Author contributions}

Study concept and design (YHG), acquisition of data (HQX, CGW, PX), drafting of the manuscript (HQX, CGW), critical revision of the manuscript for important intellectual content (YHG). All authors read and approved the final version of the manuscript.

\section{References}

[1] World Health Organization. Global hepatitis report, 2017. Available from: https://www.who.int/hepatitis/publications/global-hepatitisreport2017/en/.

[2] Rehermann B. HCV in 2015: Advances in hepatitis C research and treatment. Nat Rev Gastroenterol Hepatol 2016;13:70-72. doi: 10.1038/nrgastro. 2015.227.

[3] Chayama K, Suzuki F, Karino Y, Kawakami Y, Sato K, Atarashi T, et al. Efficacy and safety of glecaprevir/pibrentasvir in Japanese patients with chronic genotype 1 hepatitis $C$ virus infection with and without cirrhosis. J Gastroentero 2018;53:557-565. doi: 10.1007/s00535-017-1391-5.

[4] Toyoda H, Chayama K, Suzuki F, Sato K, Atarashi T, Watanabe T, et al. Efficacy and safety of glecaprevir/pibrentasvir in Japanese patients with chronic genotype 2 hepatitis C virus infection. Hepatology 2018;67:505-513. doi: 10. 1002/hep. 29510

[5] Zeuzem S, Foster GR, Wang S, Asatryan A, Gane E, Feld J], et al. Glecaprevirpibrentasvir for 8 or 12 weeks in HCV genotype 1 or 3 infection. N Engl J Med 2018;378:354-369. doi: 10.1056/NEJMoa1702417.

[6] Asselah T, Kowdley KV, Zadeikis N, Wang S, Hassanein T, Horsmans Y, et al. Efficacy of glecaprevir/pibrentasvir for 8 or 12 weeks in patients with hepatitis c virus genotype 2, 4, 5, or 6 infection without cirrhosis. Clin Gastroenterol Hepatol 2018;16:417-426. doi: 10.1016/j.cgh.2017.09.027.

[7] Asselah T, Hezode C, Zadeikis N, Elkhashab M, Colombo M, Marinho RT, et al. ENDURANCE-4: Efficacy and safety of ABT-493/ABT-530 treatment in patients with chronic HCV genotype 4, 5, or 6 infection. Hepatology 2016; 64:63A.
[8] Asselah T, Lee SS, Yao BB, Nguyen T, Wong F, Mahomed A, et al. Efficacy and safety of glecaprevir/pibrentasvir in patients with chronic hepatitis $C$ virus genotype 5 or 6 infection (ENDURANCE-5,6): an open-label, multicentre, phase 3b trial. Lancet Gastroenterol Hepatol 2019;4:45-51. doi: 10. 1016/S2468-1253(18)30341-8

[9] Forns X, Lee SS, Valdes J, Lens S, Ghalib R, Aguilar H, et al. Glecaprevir plus pibrentasvir for chronic hepatitis $C$ virus genotype $1,2,4,5$, or 6 infection in adults with compensated cirrhosis (EXPEDITION-1): a single-arm, openlabel, multicentre phase 3 trial. Lancet Infect Dis 2017;17:1062-1068. doi: 10.1016/S1473-3099(17)30496-6.

[10] Rockstroh JK, Lacombe K, Viani RM, Orkin C, Wyles D, Luetkemeyer AF, et al. Efficacy and safety of glecaprevir/pibrentasvir in patients coinfected with hepatitis $C$ virus and human immunodeficiency virus type 1 : The EXPEDITION-2 study. Clin Infect Dis 2018;67:1010-1017. doi: 10.1093/cid/ciy220.

[11] Gane E, Lawitz E, Pugatch D, Papatheodoridis G, Bräu N, Brown A, et al. Glecaprevir and pibrentasvir in patients with HCV and severe renal impairment. N Engl J Med 2017;377:1448-1455. doi: 10.1056/NEJMoa1704053.

[12] Lawitz E, Flisiak R, Abunimeh M, Sise ME, Park JY, Kaskas M, et al. Efficacy and safety of glecaprevir/pibrentasvir in renally impaired patients with chronic HCV infection. Liver Int 2020;40:1032-1041. doi: 10.1111/liv. 14320.

[13] Brown RS Jr, Buti M, Rodrigues L, Chulanov V, Chuang WL, Aguilar H, et al. Glecaprevir/pibrentasvir for 8 weeks in treatment-naïve patients with chronic HCV genotypes 1-6 and compensated cirrhosis: The EXPEDITION-8 trial. J Hepatol 2020;72:441-449. doi: 10.1016/j.jhep.2019.10.020.

[14] Poordad F, Felizarta F, Asatryan A, Sulkowski MS, Reindollar RW, Landis CS, et al. Glecaprevir and pibrentasvir for 12 weeks for hepatitis $C$ virus genotype 1 infection and prior direct-acting antiviral treatment. Hepatology 2017;66: 389-397. doi: 10.1002/hep.29081.

[15] Poordad F, Pol S, Asatryan A, Buti M, Shaw D, Hézode C, et al. Glecaprevir/Pibrentasvir in patients with hepatitis $C$ virus genotype 1 or 4 and past directacting antiviral treatment failure. Hepatology 2018;67:1253-1260. doi: 10 . 1002/hep.29671.

[16] Reau N, Kwo PY, Rhee S, Brown RS Jr, Agarwal K, Angus P, et al. Glecaprevir/pibrentasvir treatment in liver or kidney transplant patients with hepatitis C virus infection. Hepatology 2018;68:1298-1307. doi: 10.1002/hep. 30046.

[17] Dore GJ, Feld JJ, Thompson A, Martinello M, Muir AJ, Agarwal K, et al. Simplified monitoring for hepatitis $C$ virus treatment with glecaprevir plus pibrentasvir, a randomised non-inferiority trial. J Hepatol 2020;72:431440. doi: 10.1016/j.jhep.2019.10.010.

[18] Wyles D, Poordad F, Wang S, Alric L, Felizarta F, Kwo PY, et al. Glecaprevir/pibrentasvir for hepatitis $C$ virus genotype 3 patients with cirrhosis and/or prior treatment experience: A partially randomized phase 3 clinical trial. Hepatology 2018;67:514-523. doi: 10.1002/hep.29541.

[19] Gane E, Poordad F, Wang S, Asatryan A, Kwo PY, Lalezari J, et al. High efficacy of ABT-493 and ABT-530 treatment in patients with HCV genotype 1 or 3 infection and compensated cirrhosis. Gastroenterology 2016;151:651659.e1. doi: 10.1053/j.gastro.2016.07.020.

[20] A study to evaluate the efficacy and safety of glecaprevir/pibrentasvir (ABT493/ABT-530) in treatment-naive and treatment-experienced, non-cirrhotic asian adults with chronic hepatitis C. virus genotype (GT) 1 to GT6 infection with or without human immunodeficiency virus co-infection (VOYAGE-1). Available from: https://clinicaltrials.gov/ct2/show/NCT03222583? cond $=$ NCT03222583\&draw $=2 \&$ rank $=1$.

[21] Efficacy and safety of glecaprevir/pibrentasvir (ABT-493/ABT-530) in treatment-naive and treatment-experienced asian adults with chronic hepatitis $\mathrm{C}$. virus genotype (GT) 1 to GT6 infection with compensated cirrhosis and with or without human immunodeficiency virus co-infection (VOYAGE-2). Available from: https://clinicaltrials.gov/ct2/show/NCT03235349? cond $=$ NCT03235349\&draw $=2 \&$ rank $=1$.

[22] Lok AS, Sulkowski MS, Kort JJ, Willner I, Reddy KR, Shiffman ML, et al. Efficacy of glecaprevir and pibrentasvir in patients with genotype 1 hepatitis $\mathrm{C}$ virus infection with treatment failure after NS5A inhibitor plus sofosbuvir therapy. Gastroenterology 2019;157:1506-1517.e1. doi: 10.1053/j.gastro. 2019.08.008.

[23] Fontana RJ, Lens S, McPherson S, Elkhashab M, Ankoma-Sey V, Bondin M, et al. Efficacy and safety of 8 weeks of glecaprevir/pibrentasvir in treatmentnaïve, HCV-infected patients with APRI $\leq 1$ in a single-arm, open-label, multicenter study. Adv Ther 2019;36:3458-3470. doi: 10.1007/s12325-01901123-0.

[24] A study to evaluate the efficacy and safety of glecaprevir (GLE)/pibrentasvir (PIB) in treatment-naive adults in brazil with chronic hepatitis $\mathrm{C}$. virus (HCV) genotype 1-6 infection. Available from: https://clinicaltrials.gov/ct2/show/NCT03219216? cond=NCT03219216\&draw =2\&rank $=1$.

[25] McPhee F. Developments in the treatment of HCV genotype 3 infection. Expert Rev Anti Infect Ther 2019;17:775-785. doi: 10.1080/14787210. 2019.1676730.

[26] Tamori A, Inoue K, Kagawa T, Takaguchi K, Nouso K, Iwasaki Y, et al. Intention-to-treat assessment of glecaprevir + pibrentasvir combination therapy 
for patients with chronic hepatitis C in the real world. Hepatol Res 2019;49: 1365-1373. doi: 10.1111/hepr.13410.

[27] Nozaki A, Atsukawa M, Kondo C, Toyoda H, Chuma M, Nakamuta M, et al. The effectiveness and safety of glecaprevir/pibrentasvir in chronic hepatitis $C$ patients with refractory factors in the real world: a comprehensive analysis of a prospective multicenter study. Hepatol Int 2020;14:225-238. doi: 10. 1007/s12072-020-10019-z.

[28] Lampertico P, Carrión JA, Curry M, Turnes J, Cornberg M, Negro F, et al. Realworld effectiveness and safety of glecaprevir/pibrentasvir for the treatment of patients with chronic HCV infection: A meta-analysis. J Hepatol 2020;72: 1112-1121. doi: 10.1016/j.jhep.2020.01.025.

[29] Toyoda $\mathrm{H}$, Atsukawa $\mathrm{M}$, Watanabe $\mathrm{T}$, Nakamuta $\mathrm{M}$, Uojima $\mathrm{H}$, Nozaki $\mathrm{A}$, et al. Real-world experience of 12 -week direct-acting antiviral regimen of glecaprevir and pibrentasvir in patients with chronic hepatitis $C$ virus infection. J Gastroenterol Hepatol 2020;35:855-861. doi: 10.1111/jgh.14874.

[30] MAVYRET $^{\mathrm{TM}}$. Available from: https://www.accessdata.fda.gov/drugsatfda docs/label/2017/209394s000lbl.pdf.

[31] Summary of product characteristics. Available from: https://www.ema. europa.eu/en/documents/product-information/maviret-epar-product-information_en.pdf.

[32] Kosloski MP, Dutta S, Wang H, Pugatch D, Mensa F, Kort J, et al. THU-231Pharmacokinetics, safety, and tolerability of next generation direct acting antivirals ABT-493 and ABT-530 in subjects with hepatic impairment. J Hepatol 2016;64:S406.

[33] Bourliere M. Retreatment options for patients who failed direct-acting antiviral regimens containing NS5A inhibitors: Is glecaprevir/pibrentasvir a valid and robust option? Gastroenterology 2019;157:1473-1475. doi: 10.1053/j. gastro.2019.10.002

[34] Wang X, Fan X, Deng H, Zhang X, Zhang K, Li N, et al. Efficacy and safety of glecaprevir/pibrentasvir for chronic hepatitis $C$ virus genotypes 1-6 infection: A systematic review and meta-analysis. Int J Antimicrob Agents 2019;54:780-789. doi: 10.1016/j.ijantimicag.2019.07.005.

[35] EASL recommendations on treatment of hepatitis C 2018. ] Hepatol 2018; 69:461-511. doi: 10.1016/j.jhep.2018.03.026.

[36] Hepatitis C. guidance 2018 update: AASLD-IDSA recommendations for testing, managing, and treating hepatitis $C$ virus infection. Clin Infect Dis 2018;67:1477-1492. doi: 10.1093/cid/ciy585.

[37] Uemura H, Uchida Y, Kouyama JI, Naiki K, Tsuji S, Sugawara K, et al. NS5AP32 deletion as a factor involved in virologic failure in patients receiving glecaprevir and pibrentasvir. J Gastroenterol 2019;54:459-470. doi: 10. 1007/s00535-018-01543-9.

[38] Ohya K, Imamura M, Osawa M, Teraoka Y, Morio K, Fujino H, et al. Successfu retreatment with 12 weeks of glecaprevir and pibrentasvir for a genotype $2 a$ $\mathrm{HCV}$-infected hemodialysis patient who failed to respond to 8 weeks of prior glecaprevir and pibrentasvir therapy. Clin J Gastroenterol 2020;13:267270. doi: 10.1007/s12328-019-01039-w.
[39] Puoti M, Foster GR, Wang S, Mutimer D, Gane E, Moreno C, et al. High SVR12 with 8-week and 12-week glecaprevir/pibrentasvir therapy: An integrated analysis of HCV genotype 1-6 patients without cirrhosis. J Hepatol 2018 69:293-300. doi: 10.1016/j.jhep.2018.03.007.

[40] D'Ambrosio R, Pasulo L, Puoti M, Vinci M, Schiavini M, Lazzaroni S, et al. Realworld effectiveness and safety of glecaprevir/pibrentasvir in 723 patients with chronic hepatitis C. J Hepatol 2019;70:379-387. doi: 10.1016/j.jhep. 2018.11.011.

[41] Chromy D, Mandorfer M, Bucsics T, Schwabl P, Scheiner B, Schmidbauer C, et al. High efficacy of interferon-free therapy for acute hepatitis $C$ in HIVpositive patients. United European Gastroenterol J 2019;7:507-516. doi: $10.1177 / 2050640619835394$

[42] Kosloski MP, Oberoi R, Wang S, Viani RM, Asatryan A, Hu B, et al. Drug-drug interactions of glecaprevir and pibrentasvir coadministered with human immunodeficiency virus antiretrovirals. J Infect Dis 2020;221:223-231. doi: 10.1093/infdis/jiz439.

[43] Kosloski MP, Bow DAJ, Kikuchi R, Wang H, Kim EJ, Marsh K, et al. Translation of in vitro transport inhibition studies to clinical drug-drug interactions for glecaprevir and pibrentasvir. J Pharmacol Exp Ther 2019;370:278-287. doi: 10.1124/jpet.119.256966.

[44] Kosloski MP, Zhao W, Marbury TC, Preston RA, Collins MG, Pugatch D, et al. Effects of renal impairment and hemodialysis on the pharmacokinetics and safety of the glecaprevir and pibrentasvir combination in hepatitis $C$ virusnegative subjects. Antimicrob Agents Chemother 2018;62:e01990-17. doi: 10.1128/AAC.01990-17.

[45] Liu CH, Yang SS, Peng CY, Lin WT, Liu CJ, Su TH, et al. Glecaprevir/pibrentasvir for patients with chronic hepatitis $C$ virus infection and severe rena impairment. J Viral Hepat 2020;27:568-575. doi: 10.1111/jvh.13265.

[46] Ueda $Y$, Kobayashi T, Ikegami T, Miuma S, Mizuno S, Akamatsu N, et al. Efficacy and safety of glecaprevir and pibrentasvir treatment for 8 or 12 weeks in patients with recurrent hepatitis $\mathrm{C}$ after liver transplantation: Japanese multicenter experience. J Gastroenterol 2019;54:660-666. doi: 10.1007/s00535-019-01561-1.

[47] Foster GR, Asselah T, Kopecky-Bromberg S, Lei Y, Asatryan A, Trinh R, et al. Safety and efficacy of glecaprevir/pibrentasvir for the treatment of chronic hepatitis $C$ in patients aged 65 years or older. PLoS One 2019;14:e0208506. doi: $10.1371 /$ journal.pone.0208506

[48] Jonas MM, Squires RH, Rhee SM, Lin CW, Bessho K, Feiterna-Sperling C, et al. Pharmacokinetics, safety, and efficacy of glecaprevir/pibrentasvir in adolescents with chronic hepatitis $C$ virus: Part 1 of the DORA study. Hepatology 2020;71:456-462. doi: 10.1002/hep.30840

[49] Ohya K, Kawaoka T, Imamura M, Morio K, Nakahara T, Murakami E, et al. Three children treated with direct-acting antivirals for chronic hepatitis $C$ virus genotype 1b infection. Intern Med 2020;59:941-944. doi: 10. 2169/internalmedicine.3824-19. 